\title{
Kinetics of nitrobenzene biotransformation
}

\author{
A. V. N. Swamy ${ }^{1} \&$ Y. Anjaneyulu ${ }^{2}$ \\ ${ }^{I}$ Department of Chemical Engineering, JNTU College of Engineering, \\ Anantapur, India \\ ${ }^{2} J N T U$, Kukatpally, Hyderabad, India
}

\begin{abstract}
Nitrobenzene and substituted aromatics like nitro-chlorobenzene, aniline, and nitro-toluene containing chlorine, amino and methyl groups are recalcitrant in nature. These molecules are both anthropogenic and xenobiotic. Nitro benzene and nitro aromatic compounds are environmental pollutants discharged through wastewaters from nitro aromatic manufacturing plants. Nitrobenzene and other aromatics are toxic to several forms of aquatic life. However, biological transformation of nitrobenzene to non-toxic entities exists in specialized microbes, which have enzymes of aromatic catabolic pathways. Transformation of nitrobenzene is also inhibited by the presence of other toxic materials such as cyanides and sulphides that are present in industrial waste when nitrobenzene is the dominant carbonaceous material. Kinetics of the biotransformation of nitrobenzene using pure cultures isolated in the laboratory, mixture of consortium of the pooled cultures and enriched activated sludge biomass in pure substrates, mixed substrates and actual nitrobenzene plant waste has been estimated under varying input concentrations. Experimental results from these studies have been subjected to analysis by mathematical models using Monod's and Haldane's equations to test their validity in interpreting the data on inhibitory substances under stable as well as unstable state operations of wastewater treatment plants. Comparative evaluation of the kinetic parameters reveals that Monod's model can be employed for the estimation of kinetic constant $\mu$ only while Haldane's model has to be used for the calculation of $\mu_{\max }$ and $\mathrm{K}_{\mathrm{i}}$.
\end{abstract}

Keywords: kinetics, nitrobenzene, nitrochlorobenzene, and biotransformation. 


\section{Introduction}

Nitrobenzene is a substituted aromatic molecule containing nitro group. The origin of nitrobenzene to the atmosphere is due to anthropogenic activity. Anthropogenic sources are industrial wastes derived from chemical manufacturing processes such as nitro aromatic chemicals production units. Mostly the industrial wastes are originated from the wash waters of final product purification. The summary of microbial transformations of some substituted aromatic compounds together with isolated microorganisms is given in a research paper (Gibson [1]). Anaerobic degradation studies on benzene nucleus were carried out (Taylor et al. [2]) by using facultative and anaerobic microorganisms

Activated sludge process (ASP) is one of the most widely accepted biological systems for the treatment of nitro aromatic compounds. The biodegradation of aromatic pollutants under aerobic conditions was studied (Arcangeli and Arvin [3]). Existing theory suggests that operational difficulties associated with inhibitory compounds present in the waste is due to process dynamics. The significance of inhibitory mechanisms, associated with operational problems in the waste treatment, is to avoid metastable and unstable regimes. Bacteria capable of utilizing nitro aromatic compounds are found in soil and water environment. The wastewater from nitro aromatic production units predominantly contains nitrobenzene, nitrochlorobenzene, nitro toluene, and nitro cresols. Microorganisms attack these aromatics ring through a degradative pathway. The kinetics of microbial degradation of toluene were studied (Jirgensen et al. [4]). Aromatic compounds are rich in carbon content and once the rings are cleaved by enzymes the products (organic acids) enter the energy cycle. During the studies of biodegradation of nitro aromatic compounds it was observed (Hu and Sheih [5]) that nitro groups substituted by hydroxyl groups, with the elimination of the group as nitrite ion. Kinetics of the enzymatic reactions is hence significant in understanding the biotransformation of these molecules. Techniques for obtaining bio kinetic parameter values were studied (Grady [6]).

Table 1: $\quad$ Physico chemical characteristics of nitrobenzene wastewater.

\begin{tabular}{|l|l|l|}
\hline S.No & Parameter & Value \\
\hline 1 & PH & $4.5-5.6$ \\
\hline 2 & Color & Reddish brown \\
\hline 3 & C.O.D. & $1800-2200$ \\
\hline 4 & Total solids & $100-120$ \\
\hline 5 & B.O.D. & $650-800$ \\
\hline 6 & Nitrobenzene & $80-150$ \\
\hline 7 & Suspended solids & $50-80$ \\
\hline 8 & Ammonical nitrogen & $120-200$ \\
\hline 9 & Oil and Grease & $10-25$ \\
\hline
\end{tabular}

All values expressed in $\mathrm{mg} / \mathrm{L}$ except $\mathrm{pH}$ and colour. 


\section{Theory}

Evaluation of bio kinetic constants is significant for understanding the capacities of microorganisms for the operation of biological reactors. Kinetic studies on bacterial growth have given rise to considerable amount of literature. Particularly with regard to application of Monod's equation in respect of batch results.

$$
\mu=\mu_{\max }\left(\frac{S}{K_{s}+S}\right)
$$

In calculation of kinetic constants, $\mu, \mu_{\max }$, and $\mathrm{K}$ are related to reactor functions. These constants have been derived from the original MichaelisMenton's enzyme equation:

$$
v_{0}=V_{\max }\left(\frac{S}{K_{m}+S}\right)
$$

Monod's model has extensively been used for the estimation of biokinetic constants for bacterial growth on non-inhibitory substrates and for inhibitory substrates.

Nitrobenzene exhibits substrate inhibition and the fact that metastable conditions existed in the ASP lead to a review in the application of Monod's model to inhibitory growth models; resulting in the selection of Haldane's equation:

$$
\mu=\mu_{\max }\left[\frac{S}{S+K_{i}+\left(S^{2} / K_{i}\right)}\right]
$$

where $\mathrm{K}_{\mathrm{i}}$ is the inhibitor constant.

\section{Materials and method}

Pure cultures of Pseudomonas aeruginosa and Pseudomonas putida, were used in monoculture experiments. The mixed culture consisting of Pseudomonas aeruginosa and Pseudomonas putida has been used as mixed cultures in the growth responsible studies. Activated sludge biomass from a sewage treatment plant was used as the seed for plant waste studies. The seeds were then transferred into the growth medium with respective carbon sources. Specific growth rate. $\mu$ has been measured from the exponential growth phase and used in the model equations. 
The wastewater from NB plant was collected as a composite sample, transported in airtight containers, and characterized. The waste after proper dilution was sterilized with a membrane filter before it was used in the growth experiments. Compositions of the NB plant waste are given in Table 1.

\subsection{Analytical procedures}

Nitrobenzene was estimated by gas chromatography (Varian3700). Waste water was analyzed for other parameters according to standard methods APHA, AWWA, WPCF [7].Growth of biomass was measured by turbid metric method (OD at $610 \mathrm{~nm}$ ) which was then calibrated against dry weight. The cultures were incubated at a constant temperature of $30^{\circ} \mathrm{C}$.

\subsection{Experimental observations}

The synthetic waste was constituted based on proximate composition and supplemented with other essential nutrients. The specific growth rate $\mu$ derived from various initial substrate concentrations has been plotted against initial constant concentrations to derive the kinetic constants using the kinetic models. Fig. 1(a)-(d) represents the reciprocal plot deriving the kinetic constants by Monod's model while Fig. 2(a)-(d) represents the typical line of best fit by Haldane's model.

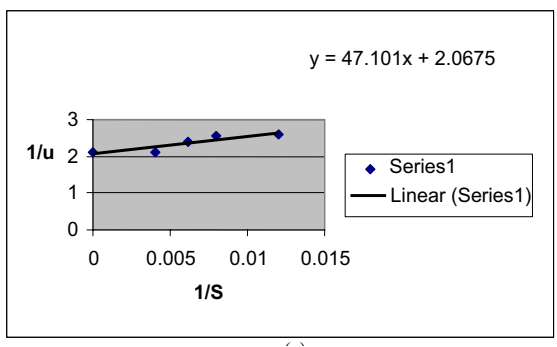

(a)

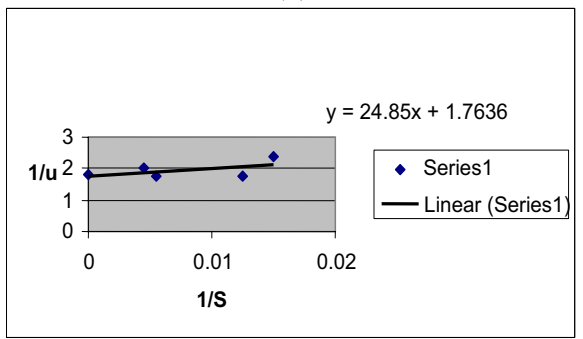

(b)

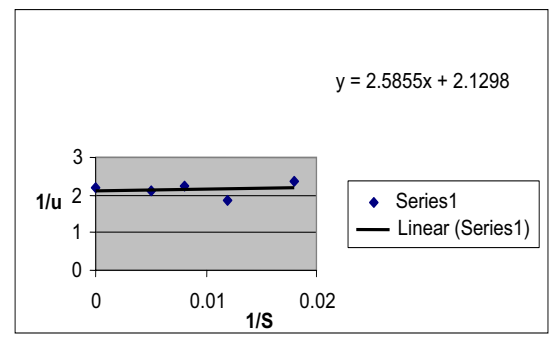

(c)

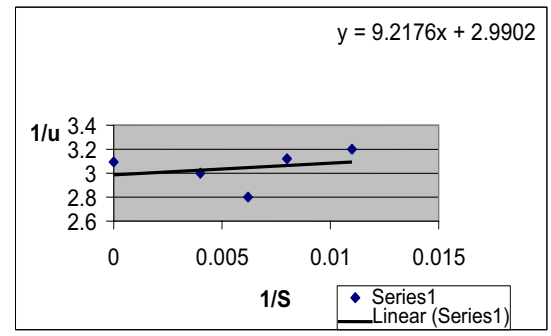

(d)

Figure 1: (a) Kinetic constants Monod model $\mathrm{K}, \mathrm{u}_{\max }, P$. aeruginosa. (b) kinetic constants Monod model $\mathrm{k}, \mathrm{u}_{\max } P$. putida, (c) kinetic constants Monod model $\mathrm{K}, \mathrm{u}_{\max }$, mixed culture synthetic waste and (d) kinetic constant Monod model $\mathrm{K}, \mu_{\max }$, mixed culture, plant waste. 


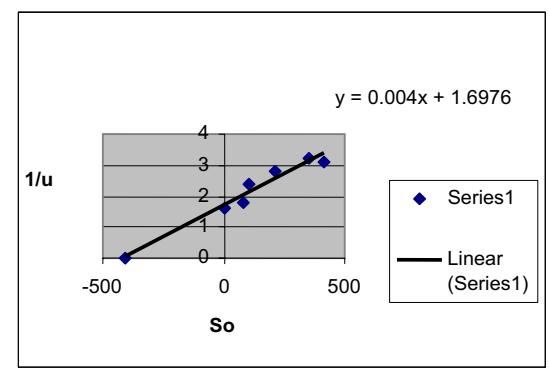

(a)

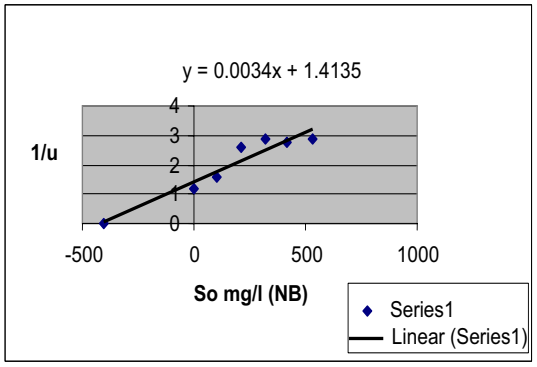

(b)

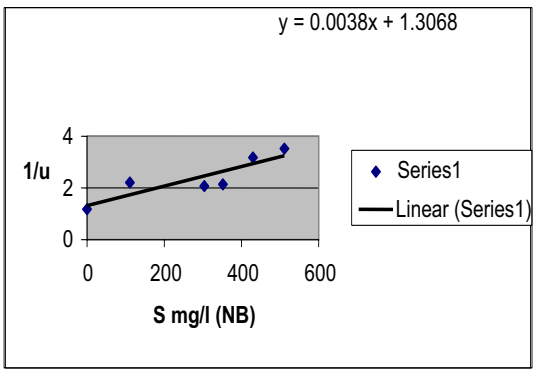

(c)

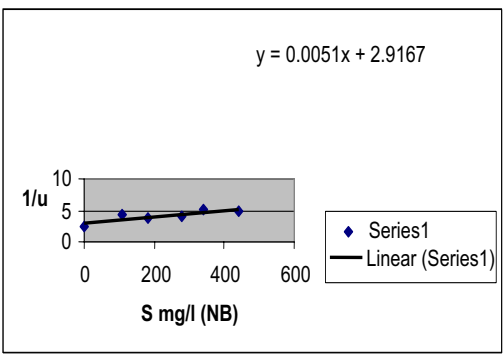

(d)

Figure 2: (a) Kinetic constant Haldane model $\mathrm{K}, \mu_{\max }$ for $P$. aeruginosa, (b) kinetic constant Haldane model $\mathrm{K}, \mu_{\max }$ for $P$. putida, (c) kinetic constants Haldane model $\mathrm{K}, \mu_{\max }$, mixed culture, synthetic waste and (d) kinetic constant Haldane Model $\mathrm{K}, \mu_{\max }$, mixed culture plant waste.

Table 2: Biokinetic constants for NB biodegradation of the values with Monod's and Haldane's models.

\begin{tabular}{|c|c|c|c|c|c|}
\hline & & Monod's & & Haldane' & lue \\
\hline S.No & Parameter & $\mu_{\max }\left(\mathrm{h}^{-1}\right)$ & $\mathrm{K}_{\mathrm{s}}\left(\mathrm{mgl}^{-1}\right)$ & $\mu_{\max }\left(\mathrm{h}^{-1}\right)$ & $\mathrm{K}_{\mathrm{i}}\left(\mathrm{mgl}^{-1}\right)$ \\
\hline 1 & $\begin{array}{l}\text { Pseudomonas } \\
\text { aeruginosa }\end{array}$ & 0.52 & 31.2 & 0.62 & 480 \\
\hline 2 & $\begin{array}{l}\text { Pseudomonas } \\
\text { putida }\end{array}$ & 0.66 & 19.5 & 0.83 & 490 \\
\hline 3 & $\begin{array}{l}\text { Mixed culture } \\
\text { with Synthetic } \\
\text { NB }\end{array}$ & 0.55 & 12.06 & 0.76 & 440 \\
\hline 4 & $\begin{array}{l}\text { Mixed culture } \\
\text { with plant } \\
\text { waste }\end{array}$ & 0.68 & 103.52 & 0.62 & 380 \\
\hline 5 & $\begin{array}{l}\text { Sewage plant } \\
\text { sludge with } \\
\text { plant waste }\end{array}$ & 0.42 & 32.25 & 0.45 & 370 \\
\hline
\end{tabular}


These expressions clearly indicate that $\mu_{\max }$ is a reaction rate constant achieved by a culture when it is grown under no limiting growth conditions. The value should then be the one that is obtained as the highest value. Thus $\mu_{\max }$ calculated by Haldane's model should be considered the true value.

Growth data from a continuously operated completely mixed activated sludge (CMAS) system has also been subjected to treatment using Monod's and Haldane's model equations. Experimental results obtained from batch and continuous culture experimentation has been subject to validation of mathematical models suggested by various investigators. The apprehensions and difficulties experienced in comparing the values obtained with synthetic media in nitrobenzene biodegradation and in actual waste water systems have been examined in the present study. One of the major difficulties experienced by previous investigators is the lack of techniques and tools in conducting pure culture experiments. Isolation, enrichment and monoculture experimentation of laboratory stored cultures have been possible in the author's laboratory to study the kinetics of biodegradation of nitrobenzene in absence and presence of secondary inhibitors. These studies have been found necessary to understand the maximum degradation rate (the maximum substrate utilization rate) of the substrate so that in the event of the absence of all environmental pressure how far the organisms can express themselves. The $\mu_{\max }$ for Pseudomonas aeruginosa of $0.62 \mathrm{hr}^{-1}$ could not be achieved when the culture is mixed with other cultures and cultivated in a defined medium along with other strains of bacteria. The $\mu_{\max }$ is further reduced when the mixed cultures are grown in actual wastewater $\left(\mu_{\max }=0.76 \mathrm{hr}^{-1}\right)$, indicating that there are several factors which influence the growth of microorganisms in a given environment. Competition for the common substrate is one of the possible reasons for a lower growth rate while noncompetitive inhibition secondary inhibitors are another. Laboratory-stored cultures are highly induced having multiple copies of plasmid genomes, which can express themselves, so that high efficiency can be achieved with such specialized seeds. Treatment of kinetic data with various model equations shows that Monod's model has limitations when used with inhibitory compounds. When the substrate induced cultures are grown at high initial substrate concentrations they tend to show lower growth rates (observations by Hill and Robinson, [8]; Gaudy et.al, [9]). This means that the organisms are under the inhibitory effect of the substrate. It is possible to achieve the exponential growth very fast and reproducibility of the experimentation is possible when the cultures are grown with initial substrate concentration is below the $K_{s}$. for experimentation on any kinetic studies with inhibitory constituents, it is necessary to obtain the $\mathrm{K}_{\mathrm{s}}$ of the system, in the first instance and then with this as the critical concentration further experiments have to be conducted to obtain value for Ks. Biodegradation can considered a feasible and reasonable system to eliminate, NB, NT from the effluents (Swamy et al [10]). 


\section{Conclusions}

Analysis of data on continuously operated reactor systems shows that under feed starve conditions $\mu_{\max }$ is much lower than the batch fed systems, indicating high detention time has to be given in the reactor. However, for the treatment of nitrobenzene, conventional ASPs with a HRT of 12 to $24 \mathrm{hrs}$ is optimum. $\mathrm{K}_{\mathrm{i}}$ of the continuously operated system is low: a situation that was postulated by Monod. The death rate of the organisms is very low which is ideal for detoxification of the toxic substrate. Engineering significance of kinetic constants is that, when hydraulic shock is effected beyond $\mu^{*}$, there is every possibility of biomass being washed out. Similarly, when organic shock is made beyond $\mathrm{K}_{\mathrm{i}}$ value the rate of degradation of the substrate is retarded, resulting in substrate build up which also reduces the treatment efficiency and functioning of the treatment plant.

\section{Acknowledgement}

Authors would like to thank M/s Hindustan Organic Chemicals Ltd., Rasayani, Dist.Raigad., Maharashtra, India, for generously funding this research work.

\section{References}

[1] Gibson, D.T. (editor), "Microbial Degradation of Organic Compounds," Marcel Decker, Inc., New York, 1986.

[2] Taylor, B. F., W.L. Campbell and T. Chinoy, "Anaerobic Degradation of the Benzene Nucleus by a Facultative Anaerobic Microorganisms." J. Bacteriol., 102(2), pp.430-437, 1970,

[3] Arcangeli, J.P. and Arvin, E., Kinetics of toluene degradation in a bio film reactor under denitrifying conditions. Wat. Sci. Tech., 29(10-11), 343-400, 1993.

[4] Jirgensen, C., Flyvbjerg J., Arvin E. and Jensen. B.K., Stoichiometry and kinetics of microbial toluene degradation under denitrifying conditions, Biodegradation 6, 147-156, 1995

[5] Hu, L.Z. and W.K. Sheih., , Anoxic bio film degradation of monocyclic aromatic compounds. Biotech. Bioengg. 30:1077-1083, 1987

[6] Grady. C.P.L., Jr., Biodegradation of Toxic Organics: Status and Potential. J. Environ. Eng., 116, 805, 1990

[7] APHA, Standard methods for the examination of water and wastewater. American Public Health Assoc., New York, N.Y., 1985.

[8] Hill G.A. and Robinson C.W, , Substrate inhibition kinetics. Phenol degradation by P.putida. Biotechnol. Bioengg 17, 1599-1615, 1975.

[9] Gaudy A.F., Rozich A.R and Gaudy E.T., Activated sludge process models for treatment of toxic and non-toxic wastes. Environ. Sci. Technol. 123-137, 1986,

[10] Swamy A.V.N and K.S.Rao, Biodegradation of Nitrobenzene and nitrotoluene by acclimated bacteria, National Journal of Life Sciences, 2(1), 2005. 